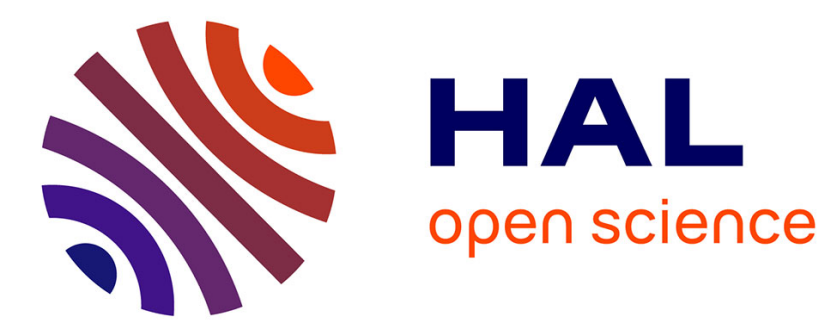

\title{
Electrovibration Modeling Analysis
}

Eric Vezzoli, Michel Amberg, Frédéric Giraud, Betty Lemaire-Semail

\section{To cite this version:}

Eric Vezzoli, Michel Amberg, Frédéric Giraud, Betty Lemaire-Semail. Electrovibration Modeling Analysis. Haptics: Neuroscience, Devices, Modeling, and Applications, Jul 2014, Paris, France. 10.1007/978-3-662-44196-1_45. hal-01253573

\section{HAL Id: hal-01253573 \\ https://inria.hal.science/hal-01253573}

Submitted on 11 Jan 2016

HAL is a multi-disciplinary open access archive for the deposit and dissemination of scientific research documents, whether they are published or not. The documents may come from teaching and research institutions in France or abroad, or from public or private research centers.
L'archive ouverte pluridisciplinaire HAL, est destinée au dépôt et à la diffusion de documents scientifiques de niveau recherche, publiés ou non, émanant des établissements d'enseignement et de recherche français ou étrangers, des laboratoires publics ou privés. 


\title{
Electrovibration modeling analysis
}

\author{
Eric Vezzoli*, Michel Amberg, Frédéric Giraud and Betty Lemaire-Semail \\ L2EP, University Lille1 F59650 Villeneuve d'Ascq, France \\ *eric.vezzoli@ed.univ-lillel.fr; \\ Michel. Amberg@univ- \\ lillel.fr; \\ frederic.giraudapolytech-lille.fr; \\ betty.semailepolytech-lille.fr
}

\begin{abstract}
Electrostatic attraction may be used to modulate the apparent friction coefficient between two surfaces. Applied to the human finger and a polarized interface, the principle can modify the user perception of the interface surface. In this paper, the different steps towards the modeling of the electrovibration phenomenon are developed. An investigation on the current modeling will be carried out, with a focus on the temporal evolution and frequency dependence of the stimulus. Thus, an improvement of the modeling will be proposed to take into account this major effect, and then, it will be checked with an experimental set-up and compared with literature results.
\end{abstract}

Keywords: Electrovibration; Electrostatic Force; Modeling; Haptic; Tactile; Friction

\section{$1 \quad$ Introduction}

The last few years have seen an increasing interest for haptic stimulation and simulation. Different technologies are available to provide a tactile feedback to a user by modifying his perception of a surface. In particular, it is possible to control the friction between a surface and a finger thanks to squeeze film effect or to electrovibration. This study is focused on the electrovibration effect: the modulation of the perceived friction coefficient due to the induced electrostatic force between a finger and a high voltage supplied plate [1]. The effect is known since the mid fifties [2], but the interest has raised only recently. Firstly, spatial division of electrode was developed to provide precise and complex stimulus pattern of conductive pads, but this solution suffered from its complexity and turned out difficult to apply [5]. Recently, electrovibration took advantage of technological improvements of fingertip's position sensor based on optical or resistive solutions. The possibility to track precisely the position of the finger leads to fine gratings simulation thanks to spatial-stimulus relation. With spatiotemporal transformation, the stimulator itself becomes easier to manufacture and it becomes possible to produce tactile feedback on transparent surfaces [1] or merge it with another tactile stimulation technique [3].

If the efficiency of the process to provide successful tactile feedback is clear, the physical modeling of the phenomenon involving the finger is not yet satisfactory. The aim of this paper is to investigate on the modeling proposed in the literature and to suggest a modification to take into account the frequency dependence of the effect. The work is organized as follows: first the experimental set up is presented, with a description of the working principle of the technology. Second, the electrostatic force applied on a finger will be described, and the 
different parameters included in the modeling will be discussed. Finally, a new modeling is proposed and the simulation results obtained will be compared with the experimental ones.

\section{$2 \quad$ Electrostatic force analysis}

\section{$2.1 \quad$ Force measurement}
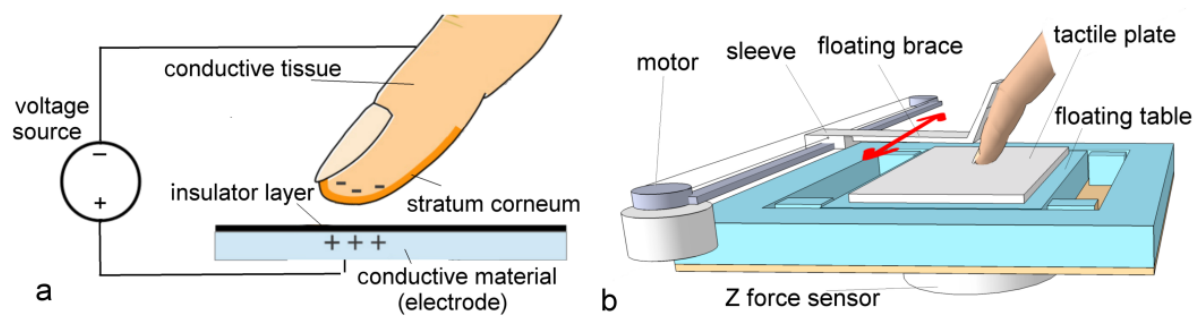

Fig. 1. a) Schematic representation of electrovibration. b) Representation of the measurement system, the finger is moved on the plate by the motor.

Electrovibration modifies the lateral force felt by the finger in motion by a modulation of the normal force. The modulation is due to electrostatic force and appears between the finger and the polarized surface (see Fig. 1.a). The experimental set-up developed and detailed in this paper is able to detect these forces.

To measure the normal and lateral force felt by the finger, a tribometer has been developed, (see Fig. 1.b). Past measurements indicate a value around $100 \mathrm{mN}$ of the induced electrostatic force [3]. A high-bandwidth sensor is needed to measure this force due to its fast varying nature. A measurement of the normal force is also required. To achieve this goal, a force sensor (9217A, KISTLER, France) is used for the normal force measurements. On top of that, a floating table equipped with a force sensor (FSG, Honywell, USA) coupled with a conditioner (max4209H, Maxim Integrated, USA) is mounted to ensure a fast acquisition of the lateral force.

To acquire the data, an oscilloscope (3014B, Tektronix, USA) is used providing both the acquisition and a visual feedback for the users. A waveform generator (33120A, Agilent, USA) provides waveforms to the high voltage amplifier supplying the tactile plate. The data are acquired and elaborated in a post-process. To provide electrostatic stimulation to the finger, an aluminum plate covered with a plastic insulator film has been used.

\subsection{Electrostatic force expression}

A finger approaching an electric field experiences an induced polarization. The polarized material interacts with an electric field generated by the electrode due to Coulomb interaction. The amplitude of this interaction is small, but is comparable with the amplitude of touchsensing action [3]. A model of the phenomenon is deduced in [4], the expression of the electrostatic force, $f_{e}$, is the following: 


$$
f_{e}=\frac{\varepsilon_{0} A v^{2}}{2\left(\frac{T_{i}}{\varepsilon_{i}}+\frac{T_{s c}}{\varepsilon_{s c}}\right)\left(T_{s c}+T_{i}\right)}
$$

where $\varepsilon_{0}$ is the vacuum permittivity, $A$ is the finger contact area, $T_{i}$ and $T_{S C}$ are the thicknesses of the insulator and the stratum corneum and $\varepsilon_{i}$ and $\varepsilon_{s c}$ are the relative permittivities of the insulator and stratum corneum. It may be noted that this expression gives as a result a constant force $f_{e}$ if the applied voltage $v$ is constant. Thanks to the experimental setup described in section 2.1, we measured the force between a finger and the polarized plate, while there is no contact between the finger and the plate. In Fig. 2 is reported the normal force measured for a given voltage signal for a floating finger over the polarized plate. We can remark oscillations due to the difficulties to maintain the finger close to the plate without any contact. But the major behavior is in agreement with (1), that is roughly a constant force once the voltage applied is constant.

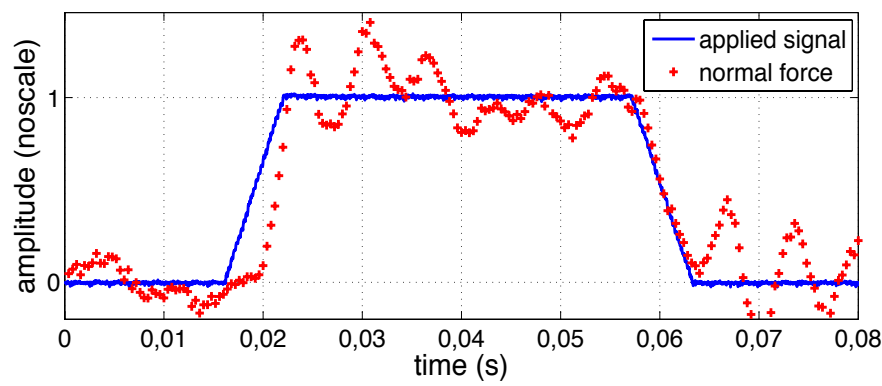

Fig. 2. Normal force for the finger suspended near the polarized plate

If we assume the force $f_{e}$ is also given by the equation (1) once the finger touches the plate, we may define the tangential force $f_{t}$ as $f_{t}=\mu\left(f_{n}+f_{e}\right)$ where $f_{n}$ is the normal force applied by the finger and $\mu$ the dynamic friction coefficient of the plate. An increase of $f_{e}$ would lead to an increase of the lateral force, creating a friction change feeling. Nevertheless, Giraud et al. [3] found an exponential reduction of the lateral force for an applied square signal when the finger is sliding on the plate. This phenomenon is unexplained by this static model. 


\section{$3 \quad$ Electrical modeling analysis}

\subsection{Frequency dependence of the parameters}

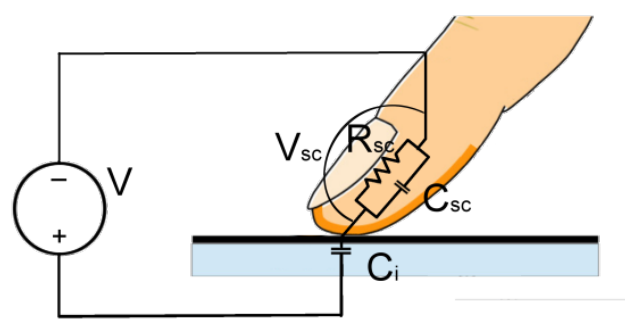

Fig. 3. Electrical representation of the stratum corneum

Every human tissue exhibits specific electrical characteristics; in this case, it is interesting to investigate the properties of the stratum corneum for its relevant role in the electrovibration force generation. The electrical properties of the stratum corneum were investigated by Yamamoto et al. [6]. In their work, the strong frequency dependence of the resistivity and permittivity is highlighted for this biological material due to its intrinsic structural characteristics. In this modeling, the resistivity of deeper tissue is neglected for its low value. On the basis of this assumption, it is possible to model the interface of the finger and the insulator like in Fig. 3, with $R_{s c}$ and $C_{s c}$ the resistance and the capacitance of the stratum corneum, $C_{i}$ the capacitance of the insulator and $v$ the applied voltage. From electrical circuit theory it comes:

$$
v_{s c}=\frac{R_{s c} C_{i} s}{1+R_{s c}\left(C_{s c}+C_{i}\right) s}=\frac{C_{i}}{C_{i}+C_{s c}} \frac{\tau s}{1+\tau s} v
$$

where $\tau=\left(C_{i}+C_{s c}\right) R_{s c}$. For a given material:

$$
C=\frac{\varepsilon_{0} \varepsilon_{r} A}{T} \quad \text { and } \quad R=\frac{\rho T}{A}
$$

where $\varepsilon_{r}$ is the material relative permittivity, $\rho$ the material resistivity, $A$ the area of the electrode and $T$ the material thickness. In this case, a plastic film has been used to insulate the finger from the electrode, leading to $C_{i}=32 \mathrm{pF}$ where $\varepsilon_{i}=3.35, T_{i}=90 \mu \mathrm{m}$ and the contact area between the finger and the plate is estimated as $A=1 \mathrm{~cm}^{2}$. For the stratum corneum, $C_{s c}=6.9 \mathrm{nF}$ with $T_{s c}=200 \mu \mathrm{m}$ [7] and $\varepsilon_{s c}=1560$ [6]. It is possible to assume $C_{s c} \gg C_{i}$ which leads, using (3), to $\tau=C_{s c} R_{s c}=\varepsilon_{0} \varepsilon_{s c} \rho_{s c}$. In Fig. 4, the frequency dependence of the time constant $\tau$ is represented; the parameters $\varepsilon_{s c}$ and $\rho_{s c}$ were measured in function of the frequency in [6]. It may be noticed that there is a strong frequency dependence of this time constant which substantially changes the behavior of (2) from a pure first order high pass filter to a more complex frequency dependence. It may be noted that this approach is valid for a static or moving finger. 


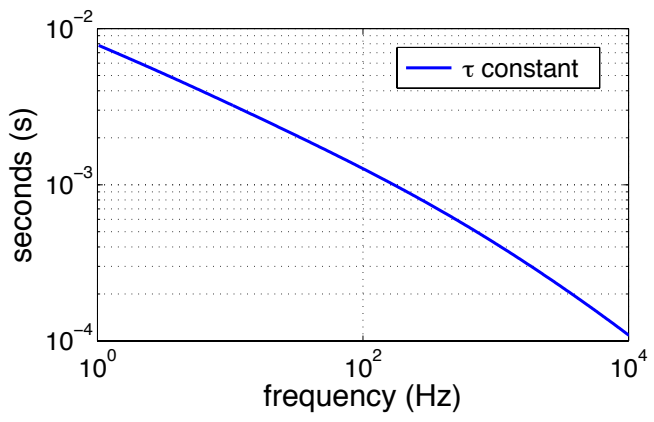

Fig. 4. $\tau$ constant frequency dependence, $\tau=\varepsilon_{0} \varepsilon_{s c} \rho_{s c}$

\subsection{Loss of charge during contact}
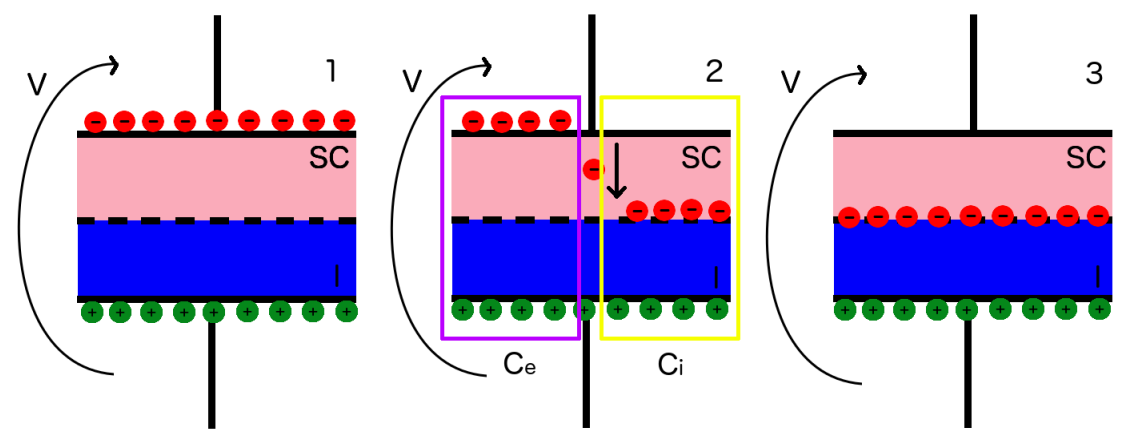

Fig. 5. Charge configuration at the border of the stratum corneum (SC) and insulator (I). The conductive part of the system is represented like the electrode of a capacitor. 1) Initial configuration on the charge when the voltage $v$ is applied. 2) Discharge through the stratum corneum with the two equivalent capacitors. 3) Final configuration of the charges after the transient.

In addition with the previous phenomenon of frequency dependence, we have to consider in the modeling the charge lost through the stratum corneum. The lost charge is gathered on the surface of the insulator as free surface charge, for this reason it no longer participates to the generation of the force on the finger, and consequently, to the measured force. This happens because the charges on the surface of the insulator are no longer mechanically bounded to the finger and the insulator sustains the induced electrostatic force. Fig. 5 illustrates three different moments of the discharge through the stratum corneum. The electrostatic force generated by $v$ associated to the first configuration is described by (1), but in the third configuration the electrostatic force between the finger and the polarized plate is 0 for the reason explained before. During the transient period represented by the second case, it is possible to imagine the applied voltage $v$, distributed across two different perfect capacitances. These capacitances are $C_{e}$ formed by the case 1 , where the charge is still on the finger, and $C_{i}$ in the case 3 , where the charge has migrated through the stratum corneum. It gives: 


$$
v=v_{e}+v_{i}=\frac{Q_{s c}}{C_{e}}+\frac{Q_{i}}{C_{i}}
$$

where $v_{e}$ and $v_{i}$ are the voltages at the terminals of the capacitors $C_{e}$ and $C_{i}$, and $Q_{s c}$ and $Q_{i}$ are respectively the charge on the stratum corneum and the insulator. The link between the two charges is determined by the rate at which the charge flows through the stratum corneum. The capacitor contributing to the electrostatic force between the finger and the insulator is $C_{e}$, because it is the only one that has a charge bounded to the finger. Then the effective part of the applied voltage contributing to the force is:

$$
v_{e}(\mathrm{~s})=\frac{Q_{s c}}{C_{e}}=\frac{C_{s c} v_{s c}}{C_{e}}=\frac{C_{s c}}{\frac{C_{i} C_{s c}}{C_{i}+C_{s c}}} \frac{C_{i}}{C_{i}+C_{s c}} \frac{\tau s}{1+\tau s} v=\frac{\tau s}{1+\tau s} v
$$

where (2) has been used. It is then possible to recover the effective force acting on the finger by replacing $v$ in (1) with $v_{e}$ to take into account the effect described above:

$$
f_{e}=\frac{\varepsilon_{0} A v_{e}^{2}}{2\left(\frac{T_{i}}{\varepsilon_{i}}+\frac{T_{s}}{\varepsilon_{s c}}\right)\left(T_{s}+T_{i}\right)}
$$

It has to be noticed that $\varepsilon_{\mathrm{sc}}$ has a frequency dependence [6] taken into account in this modeling.

\section{Simulated and experimental results}

From (6), it is possible to predict the time evolution of the lateral force $f_{t}$ for a given applied voltage signal thanks to the relationship $f_{t}=\mu\left(f_{n}+f_{e}\right)$. The evolution of the measured lateral force compared to the predicted one is proposed in Fig. 6 . The plate is polarized with a trapezium signal from 0 to $1000 \mathrm{~V}$ to investigate both the transient and the steady state. The signal is provided by an amplifier (HVA2kV, Ultravolt, USA) with a safety system to avoid any electrical shock and to limit the current to $I_{s}=250 \mu \mathrm{A}$ detailed in [3]. The mechanics of the finger and the measurement system has not been considered in this modeling.

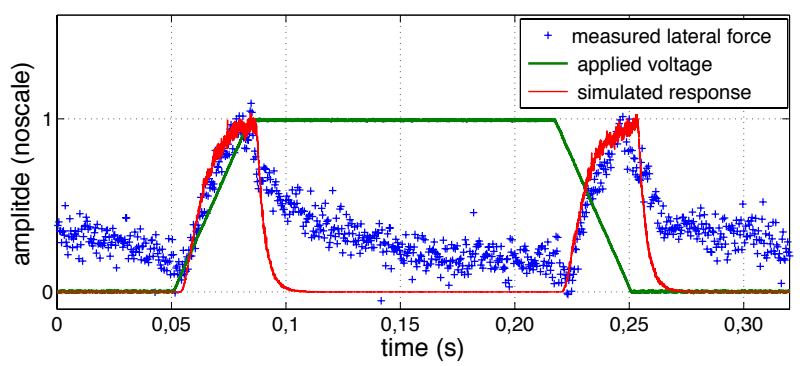


Fig. 6. Lateral force measured for the finger sliding at constant speed on the polarized plane (blue), in red is reported the modeled electrostatic force acting between the stratum corneum and the insulator.

The reported behavior has been observed on 8 different subjects, both male and female, aged from 22 to 29 . Three of them were students and the other five were from the laboratory staff, all of them gave their informed consent to perform the experiment. We can remark a very close behavior between the proposed modeling and the experimental results on the rising steps, however on the discharging parts there is a difference between the modeled and the measured signal. One assumption to explain this phenomenon could be the measurements system that can show relaxation behavior. Nevertheless the modeling is able to take into account the discharge phenomenon of the finger. The absolute value of the measured force varies from 2 to $5 \mathrm{mN}$ whereas the model, for a thickness of the stratum corneum about $0.2 \mathrm{~mm}$, predicts $2.5 \mathrm{mN}$. The variability of the force can be explained by the variability of the stratum corneum explained in [7]. It may be noticed that Giraud et al. [3] found a value of the decay of the lateral force, considering the mechanics of the measurements system, of $\tau=6 \mathrm{~ms}$ for a square signal applied. The calculated value of $\tau$ with our modeling, considering their experimental situation, is $7.7 \mathrm{~ms}$.

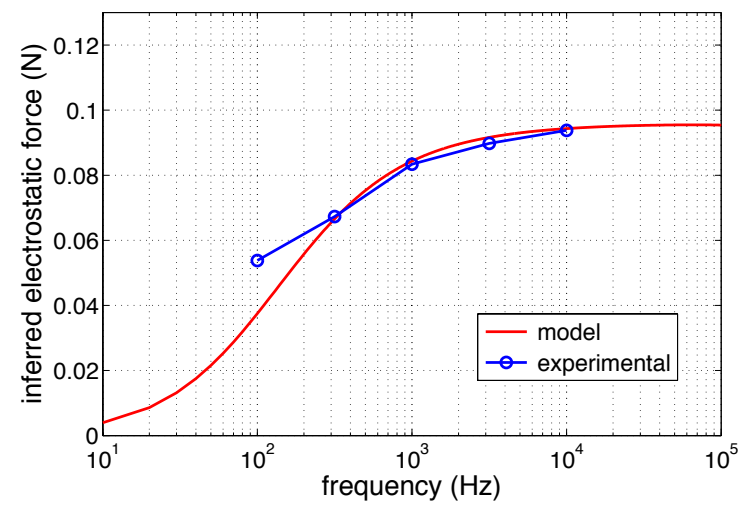

Fig. 7. Comparison of the model with experimental data taken from [5]

Another way to assess the relevance of this modeling is to compare with the experimental results from Meyer et al. [5]. In their paper, the authors investigated the frequency dependence of the electrostatic force from 100 to $10000 \mathrm{~Hz}$. The inferred measured force had a noticeable dispersion between different subjects, but generally exhibited an increasing and a successive saturation for all the measure detailed in their article. The dispersion of the force is well explained by (6), considering the variability of the thickness of the stratum corneum in human fingers reported in [7]. The proposed model in comparison with one result from [5] is reported in Fig. 7. According to the different experimental situations described in the paper, the approximation of $\tau=C_{S C} R_{S c}$ is not valid anymore for the comparable value of $C_{i}$ and $C_{S C}$ and, in the shown simulation, we considered $\tau=\left(C_{i}+C_{s c}\right) R_{s c}$. 


\section{Conclusion}

In this paper the electrovibration effect has been presented and analyzed on its temporal and frequency behavior. An electrical model has been developed to take into account the frequency dependent electrical parameters of the stratum corneum. Based on this data, the transfer function of the voltage through the stratum corneum has been calculated, and linked to the generated electrostatic force. Then the temporal response of the lateral force to a given signal has been measured and compared with the calculated one. A comparison with literature data has been carried out to assess the validity of the model.

\section{Acknowledgement}

This work was founded by the FP7 Marie Curie Initial Training Network PROTOTOUCH, grant agreement No. 317100 . The authors would like to thank the IRCICA laboratory for hosting and Clement Nadal for the useful discussion.

\section{$7 \quad$ References}

1. Bau, O., Poupyrev, I., Israr, A., Harrison, C.: TeslaTouch: electrovibration for touch surfaces. Proceedings of the 23nd annual ACM symposium on User inter- face software and technology , 283-292 (2010)

2. Mallinckrodt, E., Hughes, A.L, Sleator, W.J.: Perception by the Skin of Electrically Induced Vibrations. Science, 118, 277-278 (1953, 04)

3. Giraud, F., Amberg, M., Lemaire-Semail, B. : Merging two tactile stimulation principles: electrovibration and Squeeze Film Effect. IEEE world haptics conference, 199-203 (2013)

4. Kaczmarek, K. A., Nammi, K., Agarwal, A.K., Tyler, M.E., Haase, S.J., Beebe, D.J.: Polarity Effect in Electrovibration for Tactile Display. IEEE Transactions on Biomedical Engineering , 53 (10) 2047-2054 $(2006,10)$.

5. Meyer, D.J., Peshkin, M.A., Colgate, J.E.: Fingertip Friction Modulation due to Electrostatic Attraction. IEEE world haptics conference, 43-48 (2013)

6. Yamamoto, T., Yamamoto, Y.: Dielectrictric constant and resistivity of epidermal stratum corneum . Medical and Biological Engineering, 494-500 (1976, 09)

7. H. Fruhstorfer, Abel, U., Garthe, C-D, Knuttel, A.. Thickness of the stratum corneum of the volar fingertips . Clinical Anatomy, 429-433 (2000) 